\section{Effects of Observed Counterfactual on Prosocial Lying: A Preliminary Report}

\author{
Ryo Oda ${ }^{1, *}$, Motoki Kato ${ }^{1}$, Kai Hiraishi ${ }^{2}$ \\ ${ }^{1}$ Nagoya Institute of Technology, Nagoya 466-8555, Japan \\ ${ }^{2}$ Faculty of Letters, Keio University, Tokyo 108-8345, Japan \\ "Author for correspondence (oda.ryo@nitech.ac.jp)
}

Prosocial lie is an interesting subject in evolutionary psychology because generosity toward others is sometimes inconsistent with adherence to norms. People use justification to tell a lie without threatening the positive self-concept. Previous studies have demonstrated that observing desired counterfactuals encourages justified lying. For example, in the 'dieunder-the-cup' experimental paradigm, where no one except the participant knew the number of a rolled die, participants who rolled the die once lied less than those who rolled multiple times. This suggests that participants in the multiple-roll condition reported the highest value they observed on all rolls. However, the effects of counterfactuals were only assessed indirectly. In this study, the number that was actually rolled in the 'die-under-the-cup' paradigm was determined using a mechanical die. Participants were given an incentive of a donation to charity. Only seven of 133 participants (5.2\%) were prosocial liars (i.e., reported a larger number than their actual first roll). Six of the seven liars were female. Honesty decreased when the first roll was a lower number (1, 2 or 3$)$. Not all prosocial liars reported the largest number they had rolled.

\section{Keywords}

prosocial lie, counterfactual, justification, lying, morality

\section{Introduction}

Prosociality is an important trait of humans. Theoretical models and empirical studies have indicated that reciprocal altruism through indirect reciprocity has evolved through reputation-based partner choice (e.g., Bereczkei, Birkas, \& Kerekes, 2010; Nowak \& Sigmund, 1998). Alternatively, prosociality is a social norm, and humans tend to follow social norms and sanction departures from these norms (Chudek \& Henrich, 2011). Although these two motivations may operate simultaneously, generosity toward others is sometimes inconsistent with adherence to norms. An example is 'prosocial lie'. Using the 'die-underthe-cup' paradigm in which participants could tell a lie to behave more prosocially toward others, Lewis et al (2012) reported that people cheat more when others benefit from their unethical action. In the paradigm the die was placed under a plastic cup that had a small hole in the top, making it impossible for anyone except participants to know the number that was rolled. If the distribution of the reported numbers significantly deviated from a uniform distribution, some participants must be telling a lie. In this experiment participants were told that the experimenter's donation to a charity would be based on the numbers reported by them. Thus, participants could behave more prosocially by violating a norm (i.e., telling a lie). In fact, some English participants did so by reporting a larger number than they actually saw. This was revealed by the fact that the distribution of the reported numbers significantly deviated from a uniform distribution (Lewis et al., 2012). A similar tendency toward prosocial lying was demonstrated using a matrix task (Gino, Ayal, \& Ariely, 2013). Participants tended to over-report their performance on the task when the results were not examined, and this tendency was influenced by the number of intended beneficiaries of the unethical action. Prosocial lie is an interesting subject in evolutionary psychology of altruism. For example, Oda, Kato and Hiraishi (2015) investigated effect of watching eyes on prosocial lying using the 'die-under-the-cup' paradigm. Participants tended to tell lies that benefitted others when there were no watching eyes in the laboratory, whereas the tendency toward prosocial lying disappeared in the presence of the stylized eyes, which suggests that the watching eyes facilitated avoidance of violating norms by being honest. Factors affecting prosocial lie, however, have not been investigated in detail.

Lying is affected by situational factors, such as darkness and time to think (Halevy, Shalvi, \& Verschuere, 2013; Shalvi, Eldar, \& Bereby-Meyer, 2012; Zhong, Bohns, $\&$ Gino, 2010). One factor affecting lying is counterfactual thinking. People avoid major lies even when there is a low possibility of the lie being revealed (Fischbacher \& Heusi, 2008). Mazar, Amir and Ariely (2008) proposed that people value maintaining their positive self-concept as honest individuals; they will lie to some degree to increase their profits, but not so much as to threaten their honest self-concept. Justification is a method of lying without threatening the positive self-concept. Shalvi, Dana, Handgraaf and De Dreu (2011) argued that observing desired counterfactuals encourages justified lying, and examined this theory by employing the 'die-under-thecup' paradigm. A die was placed under a plastic cup with a small hole in the top, and only the participants knew the number of the rolled die. At the same time, the participants were asked to report the number of the first roll, which determined their reward. Participants who saw only one roll lied less than those who saw multiple rolls, suggesting that the participants in the multiple-rolls condition reported the highest value observed in the multiple rolls. Another set of participants were asked what they would have reported in randomly presented two die roll combinations 
(6 [1 $1^{\text {st }}$ roll: $\left.1-6\right] \times 6\left[2^{\text {nd }}\right.$ roll: $\left.\left.1-6\right]\right)$. Among those reporting that they would have lied, the outcome most commonly used was the desired counterfactual (the highest value they observed). From the results of two additional experiments, the authors concluded that justifications make a given dishonest act feel less unethical compared to the same dishonest act without a justification. Lewis et al. (2012) also observed an effect of counterfactuals on lying using a hypothetical three-roll experiment. Participants were given 20 combinations of three rolls of a die and asked to indicate the number rolled for payment. The results indicated that counterfactuals increased lying. A novel finding was that when no 'suitable' counterfactuals were available, outright lies were more common. However, in both studies discussed above, the investigators did not assess the actual counterfactuals that the participants had observed. In the experiment 1 of Shalvi et al. (2011), for example, the authors inferred possible effects of counterfactuals by comparing the distributions in single-roll and multiple-roll conditions with the theoretical distribution of choosing the highest value of three die rolls because the experimenter could not know actual rolled outcomes. Other experiments of Shalvi et al. (2011) and the second part of the study of Lewis et al. (2012) employed hypothetical situations by the same reason. If we could know the actual rolled outcomes by participants, however, we could gain a better understanding of the effects of counterfactuals.

In the present study, we utilized a mechanical die to investigate the number that was actually rolled in the 'dieunder-the-cup' paradigm. The die could transmit the actual number rolled to a tablet device via Bluetooth. Participants were given an incentive of a donation to charity by reporting the number of the first roll after rolling the dice three times.

\section{Methods}

Participants

A total of 226 Japanese undergraduate students (128 males and 98 females; mean age: $19.3 \pm 0.1$ years) were recruited from undergraduate psychology courses at two universities. Students participated in the study as part of their course requirement.

\section{Materials}

An interactive six-sided gaming die $(28 \mathrm{~mm}$, Dice+ Universal Board Game Controller, Game Technologies) was connected to an Android tablet (Asus Nexus 7) via Bluetooth. LED lights beneath the surface illuminated the numbers on the die. As the die was too large to roll in a cup, a cardboard box was prepared $(270 \mathrm{~mm} \times 320 \mathrm{~mm} \times$ $230 \mathrm{~mm}$; Figure 1). The lower half of one side of the box was removed, and a blackout curtain was attached in its place. A small hole was made in the top of the box to allow participants to view the die. Participants were asked to put their hand through the blackout curtain, roll the die, and observe the illuminated number through the small hole at the top. This experimental setting was used to ensure that only participants would know the result of the rolled die.

\section{Procedure}

The procedure followed that of Lewis et al. (2012). After completing a consent form, the participants were guided

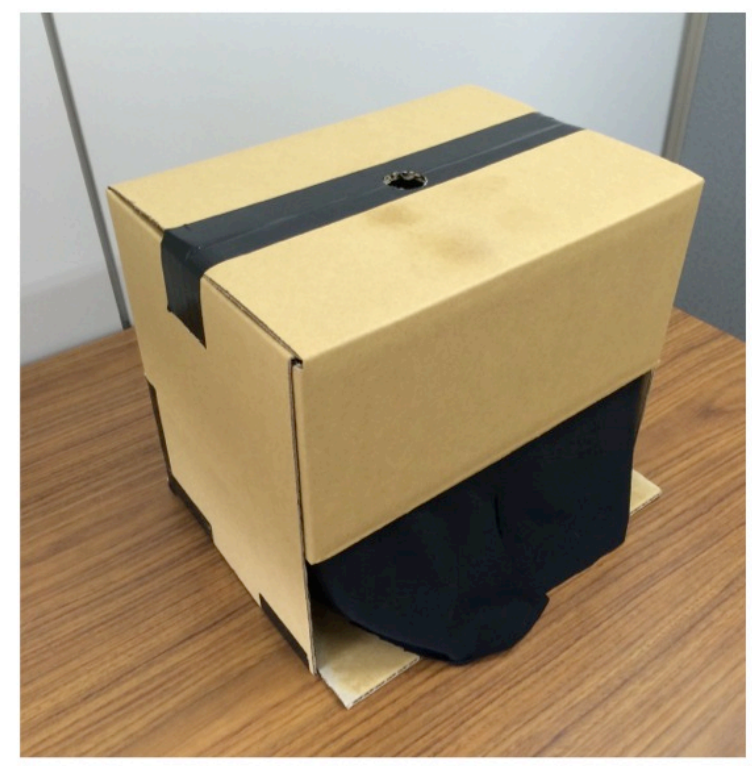

Figure 1. A cardboard box used.

to a booth surrounded by partitions. The booth contained a chair and desk, the die, the cardboard box, an instruction sheet, and a response sheet. Participants were asked to follow the procedure written on the instruction sheet, which asked them to roll the die to earn money for charity. Participants were informed that money would be donated to a non-profit organization (NPO) in support of children with intractable diseases, and the amount of the donation would be determined based on the reported value of the rolled die. For additional credibility, the booths displayed a poster of the NPO. Participants were asked to roll the die three times and observe the values through the hole in the box top. On the response sheet, participants selected the number of the first roll and identified the corresponding amount of money earned for donation (the number $\times 20 \mathrm{JPY}$ ). Participants gave the response sheet to the experimenter upon exiting the booth. Following completion of the task, participants rated their agreement, on a five-point Likert scale $(1=$ never believed, $5=$ strongly believed), to the following questions: 1) whether they believed that the researchers would donate the money generated by the rolls of the die; 2) whether they believed that, if they told a lie about the rolls of the die, their lie would be revealed; and 3) whether they believed that there was some trickery in the die. The participants were then instructed to complete the personality questionnaires.

A delayed debriefing was administered several weeks after the experiment was completed; this prevented participants from discussing the experiment with other students who had not yet participated. Charitable donations were sent to the NPO based on the numbers reported by the participants.

\section{Results}

Participants were excluded from the analysis if they did not throw the die three times; they did not complete the questionnaire; they reported that they "never believed" the researchers would donate the money; or they reported that they "strongly believed" their lie would be revealed 
and/or that there was some trickery in the die (See Table S1 of the supplemental file for the detail). As a result, 134 participants (75 males and 59 females) were analyzed (Raw data is available in the supplementary material).

Most participants reported honestly $(n=126)$, while seven participants reported a larger number (Table 1). One male participant reported a smaller number than the first roll (' 5 ' to ' 3 '). Most of the "prosocial liars" were female, though the deviation was not statistically significant (binomial test: $p=.219$ ). Of the 79 participants who rolled a lower number $(1,2$ or 3$)$ in their first throw, seven were prosocial liars. None of the 54 participants who rolled a higher number $(4,5$, or 6$)$ in their first throw was a prosocial liar. The deviation was statistically significant (Fisher's exact probability test: $p=.041$ ).

Table 1. Number of rolled in the first, second and third throws and reported.

\begin{tabular}{lcccc}
\hline Sex & \multicolumn{2}{c}{ Throw } & \multicolumn{2}{c}{ Reported } \\
\cline { 2 - 4 } & First & Second & Third & \\
\hline Male & 1 & 5 & 6 & 5 \\
Female & 2 & 5 & 6 & 6 \\
Female & 2 & 3 & 4 & 3 \\
Female & 2 & 3 & 5 & 3 \\
Female & 2 & 2 & 2 & 5 \\
Female & 3 & 4 & 3 & 4 \\
Female & 3 & 4 & 6 & 6 \\
\hline
\end{tabular}

\section{Discussion}

In our study, only $5 \%(n=7)$ of participants were prosocial liars (i.e., reported a larger number than their actual first roll), which was lower than that in the previous 'die-underthe-cup' experiment. Lewis et al. (2012) found that the participants under-reported '1's and '2's, and over-reported ' 6 's; they calculated that $9 \%$ of the participants lied that they had rolled a ' 6 ' when they had not. Considering that there might be other participants who lied that they had rolled numbers besides ' 6 ', the number of prosocial liars in their experiment should have been higher. In the control condition of Oda et al. (2015), the participants underreported '2's and ' 3 's, and over-reported ' 5 's; $11 \%$ of the participants were calculated to lie that they had rolled a ' 5 ', when they had not.

There are several possible reasons why the number of prosocial liars in the present study was lower than that reported by Lewis et al. (2012). First, socio-cultural differences between UK and Japan may have had an impact. However, a replication of the experiments of Lewis et al. (2012) in Japan revealed that the distribution of the reported numbers significantly deviated from a uniform distribution (Oda et al., 2015). Second, the use of a mechanical die, which is larger and less familiar than a common die, may have influenced the results. Indeed, we excluded participants who explicitly indicated that they "strongly believed" their lie would be revealed or that there was some trickery in the die from the analyses. The remaining participants, however, might implicitly be suspicious and reluctant to tell a lie. Third, the particular charity chosen as the beneficiary may have been impacted the results; in other words, support for children with intractable diseases may have been insufficient to trigger prosocial lying among undergraduates. In Oda et al. (2015) the participants were informed that donation would be processed through the Japanese Red Cross Society.

Six of the seven prosocial liars were female. Although the finding was not statistically significant, females may have a higher tendency to tell a prosocial lie. However, it is also possible that the donation recipient (children with intractable diseases) appealed more to females than males. The number of the first roll was ' 3 ' or lower for the seven prosocial liars and ' 4 ' or higher for all the honest participants. The deviation was statistically significant, indicating that honesty decreased when the first roll was a lower number. Additionally, six of the seven prosocial liars reported the number of their second or third roll. Though the difference was not statistically significant, it does suggest that counterfactuals have an effect on prosocial lying and supports the findings of earlier studies. It is interesting to note that not all of the prosocial liars reported the largest number they had rolled; however, we were not able to identify any clear pattern of counterfactual effects in the results. Further research into methods for evaluating prosocial lying is needed.

\section{Supplementary material}

Electronic supplementary material is available online.

\section{References}

Bereczkei, T., Birkas, B., \& Kerekes, Z. (2010). Altruism towards strangers in need: Costly signaling in an industrial society. Evolution and Human Behavior, 31, 95-103. (doi: 10.1016/j.evolhumbehav.2009.07.004)

Chudek, M., \& Henrich, J. (2011). Culture-gene coevolution, norm-psychology and the emergence of human prosociality. Trends in Cognitive Sciences, 15, 218226. (doi: 10.1016/j.tics.2011.03.003)

Fischbacher, U., \& Heusi, F. (2008). Lies in disguise, an experimental study on cheating. TWI Research Paper Series 40, Thurgau Institute of Economics, University of Konstanz. http://kops.ub.uni-konstanz.de/volltexte/2009/7461/ (accessed 11.08.18)

Gino, F., Ayal, S., \& Ariely, D. (2013). Self-serving altruism? The lure of unethical actions that benefit others. Journal of Economic Behavior and Organization, 93, 285-292. (doi: 10.1016/j.jebo.2013.04.005)

Halevy, R., Shalvi, S., \& Verschuere, B. (2013). Being honest about dishonesty: Correlating self-reports and actual Lying. Human Communication Research, 40, 54-72. (doi: 10.1111/hcre.12019)

Lewis, A., Bardis, A., Flint, C., Mason, C., Smith, N., Tickle, C., \& Zinser, J. (2012). Drawing the line somewhere: An experimental study of moral compromise. Journal of Economic Psychology, 33, 718-725. (doi: 10.1016/j.joep.2012.01.005)

Mazar, N., Amir, O., \& Ariely, D. (2008). The dishonesty of honest people: A theory of self-concept maintenance. Journal of Marketing Research, 45, 633-644. (doi: 10.1509/jmkr.45.6.633)

Nowak, M. A., \& Sigmund, K. (1998). Evolution of indirect reciprocity by image scoring. Nature, $393,573-577$. (doi: $10.1038 / 31225$ ) 
Oda, R., Kato, Y., \& Hiraishi, K. (2015). The watching-eye effect on prosocial lying. Evolutionary Psychology, 13. (doi: 10.1177/1474704915594959)

Shalvi, S., Dana, J., Handgraaf, M. J. J., \& De Dreu, C. K. W. (2011). Justified ethicality: Observing desired counterfactuals modifies ethical perceptions and behaviour. Journal of Organisational Behaviour and $\mathrm{Hu}-$ man Decision Processes, 115, 181-190. (doi: 10.1016/ j.obhdp.2011.02.001)

Shalvi, S., Eldar, O., \& Bereby-Meyer, Y. (2012). Honesty requires time (and lack of justifications). Psychological Science, 23, 1264-1270. (doi: 10.1177/0956797612443835)

Zhong, C-B., Bohns, V. K., \& Gino, F. (2010). Good lamps are the best police: Darkness increases dishonesty and self-interested behavior. Psychological Science, 21, 311-314. (doi: 10.1177/0956797609360754) 